\title{
Retinal Vessel Phenotype in Patients with a History of Retinal Vein Occlusion
}

\author{
Rachel Semecas a, b, c Louis Arnould ${ }^{d, e} \quad$ Florent Aptel $^{a, b, c} \quad$ Olivier Gavard $^{a, b, c}$ \\ Thibaud Mautuit ${ }^{a, b, c}$ Catherine Creuzot-Garcher ${ }^{d, f} \quad$ Alain Bron ${ }^{d, f}$

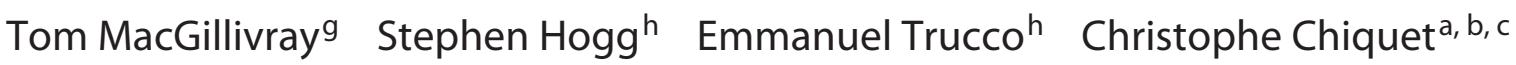 \\ ${ }^{a}$ Department of Ophthalmology, University Hospital of Grenoble, Grenoble, France; ${ }^{b}$ Grenoble Alpes University, \\ Grenoble, France; ' $\mathrm{HP} 2$ Laboratory, INSERM U1042, University Grenoble Alpes, Grenoble, France; ${ }^{\mathrm{d}}$ Department of \\ Ophthalmology, University Hospital of Dijon, Dijon, France; ${ }^{\circ}$ Clinical Epidemiology/Clinical Trials Unit, INSERM, CIC1432, \\ Dijon University Hospital, Clinical Investigation Center, Dijon, France; ${ }^{\mathrm{E} E y e}$ and Nutrition Research Group, CSGA, UMR \\ 1324 INRA, Dijon, France; 'VAMPIRE Project, Centre for Clinical Brain Sciences, University of Edinburgh, Edinburgh, UK; \\ hVAMPIRE project, Computing, School of Science and Engineering, University of Dundee, Dundee, UK
}

\section{Keywords}

Retinal vein occlusion · Retinal image analysis · Arterial diameter · Vein diameter · Fractal dimension - Tortuosity · Vessel assessment and measurement platform for images of the retina software

\begin{abstract}
Introduction: The aim of the study was to estimate the phenotype of retinal vessels using central retinal artery equivalent (CRAE), central retinal vein equivalent (CRVE), tortuosity, and fractal analysis in the unaffected contralateral eye of patients with central or branch retinal vein occlusion (CRVO or BRVO). Methods: Thirty-four patients suffering from CRVO, 15 suffering from BRVO, and 49 controlled matched subjects had a fundus image analyzed using the VAMPIRE software. The intraclass correlation coefficient and a Bland-Altman plot were done for the reproducibility study. Results: There was a lack of evidence of difference between the control group and the CRVO group for CRAE $(p=0.06), \operatorname{CRVE}(p=0.3)$, and arterio-venule ratio (AVR, $p=0.6$ ). Contralateral eyes of CRVO exhibited a significantly higher arterial and minimum arterial tortuosity values ( $p=0.012$ ), as compared with con-
\end{abstract}

karger@karger.com www.karger.com/ore

Karger!"
C 2022 The Author(s).

Published by S. Karger AG, Basel

This is an Open Access article licensed under the Creative Commons Attribution-NonCommercial-4.0 International License (CC BY-NC) (http://www.karger.com/Services/OpenAccessLicense), applicable to the online version of the article only. Usage and distribution for commercial purposes requires written permission. trol eyes. Contralateral eyes of patients with a history of BRVO had a significantly higher CRAE $(p=0.02)$, AVR ( $p=$ $0.006)$, and minimal arterial tortuosity $(p=0.05)$. Fractal analysis showed that contralateral eyes of BRVO had higher values of fractal parameters (D0a, $p=0.005$ ). Conclusion: This study suggests that CVRO or BRVO is not triggered by the same retinal vascular phenotypes in the contralateral eye. The morphology of retinal vasculature may be associated with the occurrence of RVO, independently of known risk factors.

(c) 2022 The Author(s).

Published by S. Karger AG, Basel

\section{Introduction}

Retinal vein occlusions (RVO) have prevalence rates ranging from 0.1 to $1.1 \%$ of the aging population, and bilateralization occurs in $5 \%$ of the cases over a 1 - to 3 -year period. Virchow's triad includes the 3 broad categories of factors that are thought to contribute to thrombosis, as in central retinal vein occlusion (CRVO): hypercoagulability, hemodynamic changes (stasis and turbulence), and endothelial injury/dysfunction. Two main risk factors 
have been identified: systemic hypertension and openangle glaucoma [1]. The pathogenesis of branch retinal vein occlusion (BRVO) is multifactorial in origin and not completely defined, with a possible combination of mechanical compression, degenerative changes in vessel walls, and/or hypercoagulable factors [1]. The arteriosclerotic changes (at the sites of arteriovenous crossing) are believed to result in venule occlusion through endothelial cell damage, thrombosis, and focal venous narrowing at sites of arteriovenous nicking [2].

The study of vessel caliber and tortuosity merits further consideration since it is not known whether a patient's vessel network promotes the onset of RVO, independently of association with cardiovascular risk factors. We hypothesize that the retinal phenotype (vasculature morphometry) may be associated with the occurrence of RVO. The analysis of fundus camera images offers a noninvasive measurement method to study the vascular retinal network. The vascular morphological phenotype (including tortuosity and fractal dimension) provides information on the architecture and geometry of the vessel network, which determines the efficiency of blood circulation. We used the VAMPIRE (Vessel Assessment and Measurement Platform for Images of the Retina, Universities of Edinburgh and Dundee) software, which enables a detailed quantitative analysis of the vascular morphometry [3]. VAMPIRE has been used in several studies on retina biomarkers for lacunar stroke, cognition, dementia, hypertension, and cardiovascular diseases among others [4]. More recently, VAMPIRE has been used to investigate the retinal vessel phenotype in ocular disease, such as nonarteritic ischemic optic neuropathy and glaucoma $[5,6]$.

There is evidence that vascular parameters such as central retinal vein equivalent (CRVE), central retinal artery equivalent (CRAE), arterio-venule ratio (AVR), and tortuosity are considered to be correlated between right and left eyes. We studied the vascular phenotype of the unaffected contralateral eyes of patients with RVO and compared it to a control group. Previous studies evaluated the contralateral eyes of RVO and showed the subtle abnormalities in eyes with normal appearance: late peripheral retinal leakage using peripheral fluorescein angiography [7], nonperfused foveal capillaries using adaptive optics scanning light ophthalmoscope [8], and decreased retinal nerve fiber thickness in the inferior and superior temporal quadrants using optical coherence tomography [9]. Therefore, we aimed to characterize the phenotype of retinal vessels using CRAE, CRVE, tortuosity, and fractal dimension in the nonaffected contralat- eral eye of patients with a history of CRVO and BRVO and to compare this phenotype with that of a control group matched for age, sex, systemic hypertension, and diabetes.

\section{Methods}

\section{RVO Population}

Thirty-four patients suffering from CRVO and 15 patients from BRVO were included in this multicentric case-control study in 2016 and 2017 (17 in the Ophthalmology Department of Grenoble Hospital and 22 in Dijon University Hospital). The inclusion criteria of patients were age over 18 years, diagnosis of CRVO or BRVO, and patients with ametropia (spherical equivalent) $\leq 3$ diopters (D). Exclusion criteria for the contralateral eye of the RVO eye were the presence of additional ocular diseases and fundus pictures with low quality $(n=14)$ due to cataract or poor fixation.

\section{Control Group}

Forty-nine controlled matched subjects (1:1) were prospectively included at the Ophthalmology Department of Grenoble University Hospital. The inclusion criteria were age over 18 years, patients without any ophthalmologic medical history, and patients with ametropia $\leq 3 \mathrm{D}$. Exclusion criteria were adults under guardianship or unable to consent, patients with ametropia $>3 \mathrm{D}$, and ocular disease. This control population was matched to the RVO population for age (5-year interval), sex, and systemic hypertension and diabetes.

Acquisitions and Analysis Using 30- or 45-Degree Funduscopic Color Photograph

Thirty- or 45-degree fundus camera images of the right eye were acquired, centered on the optic nerve and the macula, using a nonmydriatic camera: Visucam 200 (Carl Zeiss Meditec ${ }^{\mathrm{TM}}$, France, resolution of 2,124 $\times 2,056$ pixels) or CR2 (Canon ${ }^{\mathrm{TM}} \mathrm{Eu}$ ropa, Amstelveen, The Netherlands, resolution of $4,752 \times 3,168$ pixels).

\section{Image Analysis}

VAMPIRE measures semiautomatically morphological parameters of the retinal vessels. First, the optic disc (OD) contour and the macula center are located. This enabled the definition of the usual retinal coordinates ( $x$ axis through OD and macula centers and origin in the OD center) and circular zones around the $\mathrm{OD}$, namely, zone A (between OD center and 0.5 optic disc diameter [ODD]), zone B (between 0.5 and $1 \mathrm{ODD}$ ), and zone $\mathrm{C}$ (between 0.5 and 2 ODD), shown in Figure 1. Manual correction can be performed efficiently when the OD or fovea has been incorrectly identified automatically. Vessels are subsequently detected and labeled as arterioles or venules semiautomatically (Fig. 1).

Here, we used CRAE, the mean of the widths of the 6 largest arteries (using the revised Knudtson formulas) [10]; CRVE, as above but for the 6 largest venules [9]; AVR (=CRAE/CRVE); FD of the vascular network in zone $\mathrm{C}$, a measure of geometric complexity of the pattern of the vessels in zone $\mathrm{C}$, including the degree of branching complexity and vascular tortuosity. AVR, CRAE, and CRVE were computed in zone B and fractal measure and vascular tortuosity analysis in zone C. Raw measurements of CRAE and 

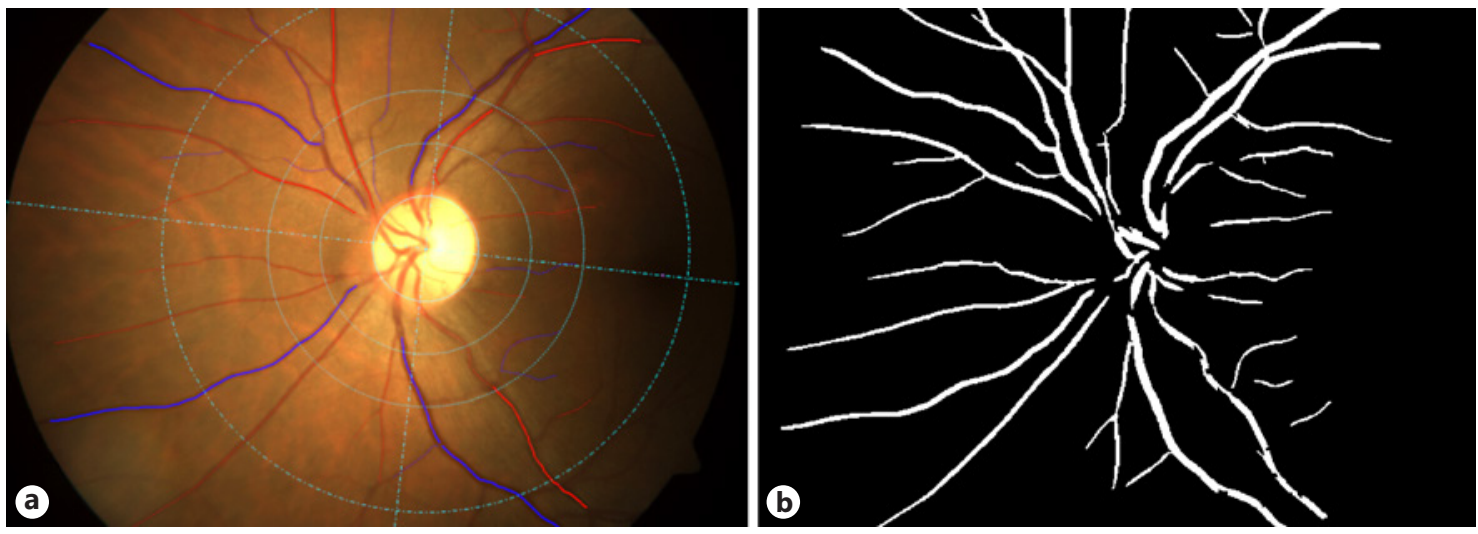

Fig. 1. Example of retinal coordinates, zones, and vasculature detection and labeling. a Example of fundus image of 1 eye of the control group and segmentation of retinal vein or artery vessels. Zones A, B, and C are defined in the standard reference system based on center and radius of the OD and on the location of the fovea. The system divides the image in concentric regions around the OD center. The zones are defined as zone A (between OD center and $0.5 \mathrm{ODD}$ ), zone $\mathrm{B}$ (between 0.5 and $1 \mathrm{ODD}$ ), and zone $\mathrm{C}$ (between 0.5 and 2 ODD). Periphery is defined as the area outside zone C. $\mathbf{b}$ Example of the VAMPIRE interface, showing a binary vessel map. OD, optic disc; ODD, optic disc diameter.

Table 1. Reproducibility study from a series of 100 fundus images

\begin{tabular}{llllllr}
\hline & $\begin{array}{l}\text { ICC } \\
\text { intraoperator } 1\end{array}$ & $95 \% \mathrm{Cl}$ & $\begin{array}{l}\text { ICC } \\
\text { intraoperator 2 }\end{array}$ & 95\% Cl & $\begin{array}{l}\text { ICC } \\
\text { interoperator }\end{array}$ \\
\hline AVR & 0.923 & $0.884-0.95$ & 0.823 & $0.730-0.883$ & 0.918 & $0.875-0.946$ \\
CRAE & 0.941 & $0.910-0.961$ & 0.914 & $0.869-0.943$ & 0.944 & $0.915-0.963$ \\
CRVE & 0.952 & $0.927-0.968$ & 0.968 & $0.952-0.979$ & 0.979 & $0.968-0.986$ \\
Arterial tortuosity & 0.969 & $0.952-0.979$ & 0.958 & $0.937-0.972$ & 0.972 & $0.957-0.981$ \\
Vein tortuosity & 0.98 & $0.970-0.987$ & 0.962 & $0.943-0.975$ & 0.992 & $0.988-0.995$ \\
\hline
\end{tabular}

AVR, arteriole-to-venule ratio; $C R A E$, central retinal artery equivalent; CRVE, central retinal vein equivalent; ICC, intraclass correlation coefficient; $\mathrm{Cl}$, confidence interval; 1, first operator; 2, second operator.

CRVE were in pixels. Tortuosity may be associated with high blood flow, angiogenesis, and blood vessel congestion.

Following previously reported studies, the pixel-to-mm conversion factor was obtained by dividing the average vertical ODD (over all images, acquired with the same camera at the same resolution) by the assumed average of the disc diameter in microns $(1,850 \mu \mathrm{m})[10-12]$. Excellent intra- and interoperator reproducibility (above 0.82 and 0.91 , respectively) for the 2 operators (R.S. and O.G.) participating in the study was obtained with 100 fundus images from healthy subjects $(n=30)$ or patients suffering from glaucoma or contralateral eyes from RVO $(n=70)$ twice by 2 different operators (R.S. and O.G.).

\section{Statistical Analysis}

Statistical analysis was performed using the Statistical Package for the Social Sciences program (SPSS 17.0 for Windows, Chicago, IL, USA). Quantitative data were expressed as mean and standard deviation, after checking the normality of data. The intraclass correlation coefficient with a confidence interval of $95 \%$ and a BlandAltman plot were done for the reproducibility study. Mean com- parisons were studied using the 2-tailed $t$ test. Statistical significance was set at $p<0.05$.

\section{Results}

\section{Reproducibility Study $(n=100)$}

The inter- and intraoperator reproducibility (ICC) was calculated from a series of 100 fundus images ( $\mathrm{Ta}$ ble 1) and was considered as excellent. Interoperator ICC ranged from 0.918 to 0.992 and intraoperator ICC from 0.823 to 0.98 .

\section{CRVO Population $(n=34)$}

This population included 22 males and $41 \%$ had systemic hypertension. The vascular parameters of the contralateral eye were compared with the control eyes (Ta- 
Table 2. Vascular parameters of the population with a history of CRVO, compared to the control group

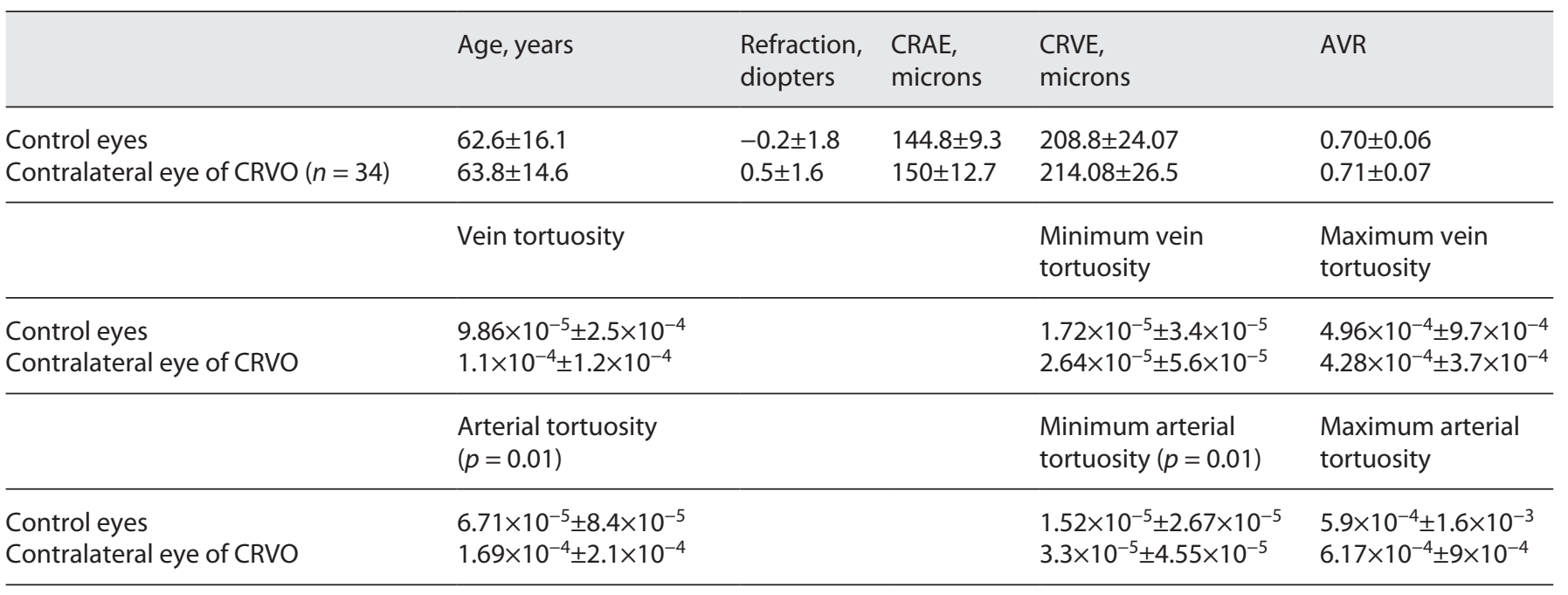

The results are expressed as the mean \pm SD. CRVO, central retinal vein occlusion; AVR, arteriole-to-venule ratio; CRAE, central retinal artery equivalent; $C R V E$, central retinal vein equivalent.

Table 3. Fractal dimensions of the arterial vasculature of the population with a history of CRVO, compared to the control group

\begin{tabular}{|c|c|c|c|c|c|c|c|c|c|}
\hline & $\mathrm{DOa}$ & D1a & $\mathrm{D} 2 \mathrm{a}$ & DOv & D1v & $\mathrm{D} 2 \mathrm{v}$ & D0tot & D1tot & D2tot \\
\hline Control eyes & $1.56 \pm 0.07$ & $1.55 \pm 0.07$ & $1.55 \pm 0.07$ & $1.53 \pm 0.06$ & $1.52 \pm 0.06$ & $1.51 \pm 0.06$ & $1.72 \pm 0.05$ & $1.71 \pm 0.05$ & $1.71 \pm 0.05$ \\
\hline Contralateral eye of CRVO & $1.57 \pm 0.08$ & $1.56 \pm 0.08$ & $1.56 \pm 0.08$ & $1.54 \pm 0.08$ & $1.53 \pm 0.08$ & $1.53 \pm 0.08$ & $1.74 \pm 0.07$ & $1.73 \pm 0.07$ & $1.72 \pm 0.07$ \\
\hline
\end{tabular}

There was no statistically significant difference between the 2 groups for fractal dimension analysis. The results are expressed as the mean \pm SD. CRVO, central retinal vein occlusion; D0, capacity dimension; D1, information dimension; D2, correlation dimension; A, arterial; $\mathrm{V}$, venule; Tot, total.

bles 2,3). No significant difference was found between the control group and the CRVO group for age $(p=0.75)$, refraction $(p=0.08)$, CRAE $(p=0.06)$, CRVE $(p=0.3)$, and $\operatorname{AVR}(p=0.6)$. Contralateral eyes of CRVO exhibited a significantly higher arterial and minimum arterial tortuosity values $(p=0.012)$. There was no statistically significant difference between the 2 groups for fractal dimension analysis $(p=0.26)$.

\section{BRVO Population $(n=15)$}

This population included 8 males and $35 \%$ had systemic hypertension. The vascular parameters of the contralateral eye of 15 patients were compared with a group of 15 matched control eyes (Tables 4, 5). Contralateral eyes of patients with a history of BRVO had a higher CRAE $(p=0.02)$, AVR $(p=0.006)$, and minimal arterial tortuosity $(p=0.05)$. Fractal analysis showed that contra- lateral eyes of BRVO had significantly higher values of fractal dimension, such as D0a $(p=0.005)$, D1a $(p=$ $0.008)$, D2a $(p=0.008)$, D0tot $(p=0.01)$, D1tot $(p=0.01)$, and D2tot $(p=0.01)$.

\section{Discussion}

This original study showed that contralateral eyes exhibit (a) in cases of CRVO, a greater arterial tortuosity values, and (b) in cases of BRVO, a greater CRAE, AVR, arterial tortuosity, and higher values of fractal parameters, as compared with the control group matched for age, sex, systemic hypertension, and diabetes. These exploratory results strongly suggest that the morphology of the retinal vasculature may be associated with the occurrence of RVO, independent of known risk factors. 
Table 4. Vascular parameters of the population with a history of BRVO and the control population

\begin{tabular}{|c|c|c|c|c|c|}
\hline Control eyes & $61.87 \pm 13.3$ & $-0.6 \pm 1.9$ & $149.3 \pm 12.4$ & $216.3 \pm 23.5$ & $0.69 \pm 0.06$ \\
\hline \multirow[t]{2}{*}{ Contralateral eye of BRVO $(n=15)$} & $64.47 \pm 13.56$ & $0.6 \pm 1.5$ & $166.7 \pm 25.6$ & $219.2 \pm 20.8$ & $0.76 \pm 0.05$ \\
\hline & Vein tortuosity & & & $\begin{array}{l}\text { Minimum vein } \\
\text { tortuosity }\end{array}$ & $\begin{array}{l}\text { Maximum vein } \\
\text { tortuosity }\end{array}$ \\
\hline Control eyes & $8.08 \times 10^{-5} \pm 1.6 \times 10^{-4}$ & & & $1.22 \times 10^{-5} \pm 2.5 \times 10^{-5}$ & $1.02 \times 10^{-3} \pm 3.37 \times 10^{-3}$ \\
\hline \multirow[t]{2}{*}{ Contralateral eye of BRVO } & $8.08 \times 10^{-5} \pm 8.77 \times 10^{-5}$ & & & $2.4 \times 10^{-5} \pm 4.3 \times 10^{-5}$ & $1.58 \times 10^{-3} \pm 4.9 \times 10^{-3}$ \\
\hline & Arterial tortuosity & & & $\begin{array}{l}\text { Minimum arterial } \\
\text { tortuosity }(p=0.05)\end{array}$ & $\begin{array}{l}\text { Maximum arterial } \\
\text { tortuosity }\end{array}$ \\
\hline
\end{tabular}

The results are expressed as the mean $\pm S D$. AVR, arteriole-to-venule ratio; $C R A E$, central retinal artery equivalent; $C R V E$, central retinal vein equivalent; BRVO, branch retinal vein occlusion.

Table 5. Fractal dimensions of the arterial vasculature

\begin{tabular}{llllllllll}
\hline & $\begin{array}{l}\text { D0a } \\
(p=0.005)\end{array}$ & $\begin{array}{l}\text { D1a } \\
(p=0.008)\end{array}$ & $\begin{array}{l}\text { D2a } \\
(p=0.008)\end{array}$ & D0v & D1v & D2v & $\begin{array}{l}\text { D0tot } \\
(p=0.01)\end{array}$ & $\begin{array}{l}\text { D1tot } \\
(p=0.01)\end{array}$ & $\begin{array}{l}\text { D2tot } \\
(p=0.01)\end{array}$ \\
\hline Control eyes & $1.55 \pm 0.06$ & $1.55 \pm 0.06$ & $1.55 \pm 0.06$ & $1.52 \pm 0.06$ & $1.51 \pm 0.06$ & $1.51 \pm 0.06$ & $1.71 \pm 0.05$ & $1.71 \pm 0.05$ & $1.70 \pm 0.05$ \\
Contralateral eye of BRVO & $1.63 \pm 0.07$ & $1.62 \pm 0.07$ & $1.61 \pm 0.07$ & $1.53 \pm 0.07$ & $1.52 \pm 0.07$ & $1.52 \pm 0.07$ & $1.76 \pm 0.05$ & $1.76 \pm 0.05$ & $1.75 \pm 0.05$ \\
\hline
\end{tabular}

D0, capacity dimension; D1, information dimension; D2, correlation dimension; A, arterial; V, venule; Tot, total.

\section{Patients with a CRVO History}

Epidemiological studies have shown that contralateral eyes of patients with a history of CRVO in 1 eye have (a) a significantly increased risk of developing RVO compared with the general population (7\% probability within 4 years) $[13,14]$ and (b) an increased number of nonperfused capillaries near the foveal avascular zone and decreased perfused foveal microvascular density [8]. Therefore, studying contralateral eyes may elucidate the early pathological changes signaling a future occlusive event.

The significant finding in our CRVO population was the abnormal higher arterial tortuosity in patients with a history of CRVO. This abnormality could increase the blood resistance and modify the blood rheology in some retinal vessels and may indicate a predisposition for occurrence of RVO. In the literature, reduced arterial tortuosity is usually reported with age, hypertension, and BMI, whereas increased venular tortuosity is associated with a younger age, higher BP, and lower HDL [15].
The other important finding was the absence of changes of CRAE, CRVE, AVR, and fractal dimension in patients as compared with the matched control group. Notwithstanding the pilot size of our cohort, this could be a major finding since these parameters are correlated with age, BMI, and history of hypertension [16], which were all taken into account with matching populations.

\section{Patients with a BRVO History}

Patients with a history of BRVO had higher CRAE and AVR, whereas CRVE was not significantly different when compared with the control group. A larger arteriolar caliber has been previously reported with current cigarette smoking [17], the activity of plasma GPx-3, a major player in oxidative stress regulation [17], calcium channel blockers, combined use of aspirin and antihypertensive agents [18], and higher levels of serum glucose (glycated hemoglobin HbA1c) [18]. These factors should be therefore considered in future studies. In the present study, 
only 6 patients out of 49 took calcium channel blockers ( 4 CRVO and 2 BRVO).

The increase of AVR is probably due to the significant increase in CRAE, whereas CRVE is comparable in both groups. On the other hand, matching both groups with the presence or absence of hypertension may explain why we did not measure the well-known decrease in CRAE associated with hypertension $[19,20]$. One previous study investigated the vascular phenotype of eyes $(n=25)$ before development of BRVO and found that AVR was significantly smaller in eyes, whereas CRAE and CRVE did not vary significantly as compared with the fellow eyes which did not develop BRVO. AVR of the contralateral eye of BRVO in our study $(0.76 \pm 0.05)$ was close to that found (0.73) in fellow eyes in the study of Kawasaki et al. [21]. On the other hand, severe arteriovenous nicking, isolated retinopathy, and a smaller angle at the crossing site were more prevalent in eyes which developed BRVO compared with fellow eyes. Our study is complementary to this latter study since we studied only fellow eyes at the time of BRVO, which is different from a longitudinal study. We also compared the fellow eyes to a control group matched for hypertension, age, and diabetes.

We found that contralateral eyes of BRVO have higher fractal dimension of the vasculature network. The FD index quantifies the complexity and density of the vessel branching pattern visible in a fundus image. FD variations are an indicator of deviations from the normal or optimized network and, when reduced, a potential marker of cardiovascular (coronary disease) or neurologic (stroke) disease. Our results suggest that these patients maintain a high level of complexity even if hypertension and age are considered.

We did not find an overall increase in tortuosity, whereas the minimal arterial tortuosity was increased significantly in these patients. This suggests a change in the distribution of arterial tortuosity among arterioles within unaffected contralateral eyes in both CRVO and BRVO populations. This difference is not likely associated with diabetes and systemic hypertension since these factors were well balanced with the control group. Factors known to have an impact on tortuosity were not significantly involved in our series (refraction and race). The significance of this result should be confirmed in further studies and other associated factors should be investigated, such as cardiovascular diseases (such as ischemic heart disease [16], obesity [15], dyslipidemia [22], and renal dysfunction [23]).

Strengths of this study were (a) the excellent inter- and intraobserver agreement (above 0.9 and 0.8 , respectively) using VAMPIRE, consistent with that described in other studies using this software $[24,25]$, and (b) matching of the control group on potential confusion factors (hypertension, age, and diabetes). This control group was matched not only for well-known factors influencing vascular parameters (age) [15] and systemic hypertension but also for risk factors of RVO (diabetes and systemic hypertension) [1]. No RVO subject in our cohort had glaucoma.

We acknowledge several limitations of this pilot study. First, the modest (pilot size) size of the cohort. Second, the absence of measurements of blood pressure or the assessment of the severity of hypertension. Many studies reported association between lower monofractal dimensions and higher mean arterial blood pressure [26, 27]. Association between lower fractal dimensions and diastolic blood pressure was also reported $[26,27]$. Other clinical variables such as history of cardiovascular or neurovascular disease, BMI, and dyslipidemia may alter the retinal vascular phenotype in the RVO and control groups [28].Third, the use of images taken at different resolutions. To partially correct for these magnification differences between cameras, a calibration factor was obtained by measuring the average vertical height of the OD (in pixels) in all retinal images with the same resolution and the same camera and dividing the assumed average of the disc diameter $(1,850 \mu \mathrm{m}$, average disc diameter measured for the Caucasian subjects) with the average value ODD measurements in pixels in the same series. This technique has been extensively used in different studies using SIVA [12] and IVAN [29]. Fourth, associations between smaller retinal vessel diameters and longer axial length and more myopic refraction were reported previously [30, 31]. The effect of refraction was limited in our study since the spherical equivalent was $0.5 \pm 1.5 \mathrm{D}$ in the RVO group and $0.5 \pm 1.5 \mathrm{D}$ in the control group. Ideally, it would be useful to measure axial length in order to convert pixels into microns using Bennet's formula [32].

Fifth and finally, we assumed that contralateral eyes reflected the phenotype of both eyes. We based this hypothesis on previous studies showing good correlations for vascular parameters between right and left eyes. Using VAMPIRE (Kirin, Mirna: Genetic Analysis of Retinal Traits. University of Edinburgh, Ph Thesis, 2013, https:// era.ed.ac.uk/handle/1842/9619?show=full), monofractal and multifractal dimensions of the retinal vasculature were also tested for left and right eye correlation $(n=130$ images of healthy eyes). Correlation values were higher compared to the branching parameters. Left-right eye correlations were 0.78 for monofractal dimensions and 
0.50 for multifractal dimensions. We note however that the degree of symmetry of the morphology and its quantitative characterization are the object of current discussion [33].

\section{Conclusion}

This pilot study in patients with a history of CVRO or BRVO suggests that both diseases are not associated with the same retinal vascular phenotypes in the contralateral eye. Contralateral eyes of CRVO eyes exhibited a higher tortuosity, whereas contralateral eyes of BRVO eyes had higher CRAE, AVR, and fractal dimension. These factors should be studied further in a longitudinal, larger study to better understand the potential risk factors associated with the retinal phenotype.

\section{Statement of Ethics}

Written informed consent was obtained from the subjects after explanation of the experiment. The study followed the Declaration of Helsinki guidelines for research involving human subjects and was approved by the local Institutional Review Board (IRB\# 5921).

\section{Conflict of Interest Statement}

C. Chiquet: Thea, Allergan, and Horus. A. Bron: Aerie, Allergan, Bausch Lomb, Santen, and Théa. F. Aptel: Aerie, Allergan, BaushLomb, Glaukos, Horus, Quantel, Santen, and Thea. C. Creuzot-Garcher: Allergan, Bayer, Alcon, Novartis, Roche, Théa, and Bausch and Lomb. R. Semecas, L. Arnould, O. Gavard, T. Mautuit, T. MacGillivray, S. Hogg, and E. Trucco: none.

\section{Funding Sources}

The study was funded by the Association de Recherche et de Formation en Ophtalmologie (ARFO) and Fondation de France (Berthe Fouassier grant).

\section{Author Contributions}

All authors made substantial contributions to the conception or design of the work; or the acquisition, analysis, or interpretation of data for the work; drafting the work or revising it critically for important intellectual content; final approval of the version to be published; they agree to be accountable for all aspects of the work in ensuring that questions related to the accuracy or integrity of any part of the work are appropriately investigated and resolved.

\section{References}

1 Hayreh SS. Prevalent misconceptions about acute retinal vascular occlusive disorders. Prog Retin Eye Res. 2005 Jul;24(4):493-519.

2 Koch E, Rosenbaum D, Brolly A, Sahel JA, Chaumet-Riffaud P, Girerd X, et al. Morphometric analysis of small arteries in the human retina using adaptive optics imaging: relationship with blood pressure and focal vascular changes. J Hypertens. 2014 Apr;32(4): $890-8$.

3 Giachetti A, Ballerini L, Trucco E. Accurate and reliable segmentation of the optic disc in digital fundus images. J Med Imaging. 2014 Jul;1(2):024001.

4 McGrory S, Cameron JR, Pellegrini E, Warren C, Doubal FN, Deary IJ, et al. The application of retinal fundus camera imaging in dementia: a systematic review. Alzheimers Dement. 2017;6:91-107.

5 Remond P, Aptel F, Cunnac P, Labarere J, Palombi K, Pepin JL, et al. Retinal vessel phenotype in patients with nonarteritic anterior ischemic optic neuropathy. Am J Ophthalmol. 2019;208:178-84.

6 Chiquet C, Gavard O, Arnould L, Mautuit T, Macgillivray TJ, Bron AM, et al. Retinal vessel phenotype in patients with primary open-angle glaucoma. Acta Ophthalmol. 2020 Feb; 98(1):e88-93
7 Tsui I, Bajwa A, Franco-Cardenas V, Pan CK, Kim HY, Schwartz SD. Peripheral fluorescein angiographic findings in fellow eyes of patients with branch retinal vein occlusion. Int J Inflam. 2013;2013:464127.

8 Pinhas A, Dubow M, Shah N, Cheang E, Liu $\mathrm{CL}$, Razeen M, et al. Fellow eye changes in patients with nonischemic central retinal vein occlusion: assessment of perfused foveal microvascular density and identification of nonperfused capillaries. Retina. 2015 Oct;35(10): 2028-36.

9 Kim S, Sung KR, Joe SG, Kim JT, Lee DH, Lee JY, et al. Comparison between glaucomatous and non-glaucomatous eyes with unilateral retinal vein occlusion in the fellow eye. Korean J Ophthalmol. 2013 Dec;27(6):440-5.

10 Knudtson MD, Lee KE, Hubbard LD, Wong TY, Klein R, Klein BE. Revised formulas for summarizing retinal vessel diameters. Curr Eye Res. 2003 Sep;27(3):143-9.

11 Varma R, Tielsch JM, Quigley HA, Hilton SC, Katz J, Spaeth GL, et al. Race-, age-, gender-, and refractive error-related differences in the normal optic disc. Arch Ophthalmol. 1994 Aug;112(8):1068-76.
12 Hubbard LD, Brothers RJ, King WN, Clegg LX, Klein R, Cooper LS, et al. Methods for evaluation of retinal microvascular abnormalities associated with hypertension/sclerosis in the Atherosclerosis Risk in Communities Study. Ophthalmology. 1999 Dec;106(12): 2269-80.

13 Natural history and clinical management of central retinal vein occlusion. The Central Vein Occlusion Study Group. Arch Ophthalmol. 1997 Apr;115(4):486-91.

14 McIntosh RL, Rogers SL, Lim L, Cheung N, Wang JJ, Mitchell P, et al. Natural history of central retinal vein occlusion: an evidencebased systematic review. Ophthalmology. 2010 Jun;117(6):1113-23.e15.

15 Cheung CY, Zheng Y, Hsu W, Lee ML, Lau QP, Mitchell P, et al. Retinal vascular tortuosity, blood pressure, and cardiovascular risk factors. Ophthalmology. 2011 May;118(5): 812-8.

16 Witt N, Wong TY, Hughes AD, Chaturvedi $\mathrm{N}$, Klein BE, Evans R, et al. Abnormalities of retinal microvascular structure and risk of mortality from ischemic heart disease and stroke. Hypertension. 2006 May;47(5):97581. 
17 Daien V, Carriere I, Kawasaki R, Cristol JP, Villain M, Fesler P, et al. Retinal vascular caliber is associated with cardiovascular biomarkers of oxidative stress and inflammation: the POLA study. PLoS One. 2013;8(7):e71089.

18 Wong TY, Islam FM, Klein R, Klein BE, Cotch MF, Castro C, et al. Retinal vascular caliber, cardiovascular risk factors, and inflammation: the multi-ethnic study of atherosclerosis (MESA). Invest Ophthalmol Vis Sci. 2006 Jun;47(6):2341-50.

19 McGowan A, Silvestri G, Moore E, Silvestri V, Patterson CC, Maxwell AP, et al. Evaluation of the retinal vasculature in hypertension and chronic kidney disease in an elderly population of irish nuns. PLoS One. 2015;10(9): e0136434.

20 Triantafyllou A, Doumas M, Anyfanti P, Gkaliagkousi E, Zabulis X, Petidis K, et al. Divergent retinal vascular abnormalities in normotensive persons and patients with never-treated, masked, white coat hypertension. Am J Hypertens. 2013 Mar;26(3):318-25.

21 Kawasaki R, Nagano E, Uno M, Okada M, Kawasaki Y, Kitamura A. Retinal vascular features associated with risk of branch retinal vein occlusion. Curr Eye Res. 2013 Sep;38(9): 989-93.

22 Owen CG, Rudnicka AR, Nightingale CM, Mullen R, Barman SA, Sattar N, et al. Retinal arteriolar tortuosity and cardiovascular risk fac- tors in a multi-ethnic population study of 10-year-old children; the Child Heart and Health Study in England (CHASE). Arterioscler Thromb Vasc Biol. 2011 Aug;31(8):1933-8.

23 Sasongko MB, Wong TY, Donaghue KC, Cheung N, Jenkins AJ, Benitez-Aguirre P, et al. Retinal arteriolar tortuosity is associated with retinopathy and early kidney dysfunction in type 1 diabetes. Am J Ophthalmol. 2012 Jan;153(1):176-83.e1.

24 MacGillivray TJ, Cameron JR, Zhang Q, ElMedany A, Mulholland C, Sheng Z, et al. Suitability of UK biobank retinal images for automatic analysis of morphometric properties of the vasculature. PLoS One. 2015;10(5): e0127914.

25 Perez-Rovira A, MacGillivray T, Trucco E, Chin KS, Zutis K, Lupascu C, et al. VAMPIRE: vessel assessment and measurement platform for images of the REtina. Annu Int Conf IEEE Eng Med Biol Soc. 2011;2011:3391-4.

26 Cheung CY, Thomas GN, Tay W, Ikram MK, Hsu W, Lee ML, et al. Retinal vascular fractal dimension and its relationship with cardiovascular and ocular risk factors. Am J Ophthalmol. 2012 Oct;154(4):663-74e1.

27 Kurniawan ED, Cheung N, Cheung CY, Tay WT, Saw SM, Wong TY. Elevated blood pressure is associated with rarefaction of the retinal vasculature in children. Invest Ophthalmol Vis Sci. 2012 Jan 31;53(1):470-4.
28 Liew G, Wang JJ, Mitchell P, Wong TY. Retinal vascular imaging: a new tool in microvascular disease research. Circ Cardiovasc Imaging. 2008 Sep;1(2):156-61.

29 Moradi A, Sepah YJ, Ibrahim MA, Sophie R, Moazez C, Bittencourt MG, et al. Association of retinal vessel calibre and visual outcome in eyes with diabetic macular oedema treated with ranibizumab. Eye. 2014 Nov;28(11): 1315-20.

30 Patton N, Maini R, MacGillivary T, Aslam TM, Deary IJ, Dhillon B. Effect of axial length on retinal vascular network geometry. Am J Ophthalmol. 2005 Oct;140(4): 648-53.

31 Lim LS, Cheung CY, Lin X, Mitchell P, Wong TY, Mei-Saw S. Influence of refractive error and axial length on retinal vessel geometric characteristics. Invest Ophthalmol Vis Sci. 2011 Feb;52(2):669-78.

32 Bennett AG, Rudnicka AR, Edgar DF. Improvements on Littmann's method of determining the size of retinal features by fundus photography. Graefes Arch Clin Exp Ophthalmol. 1994 Jun;232(6):361-7.

33 Cameron JR, Megaw RD, Tatham AJ, McGrory S, MacGillivray TJ, Doubal FN, et al. Lateral thinking: interocular symmetry and asymmetry in neurovascular patterning, in health and disease. Prog Retin Eye Res. 2017; 59:131-57. 